\title{
Changes of lidocaine concentration and physiological indices in dogs during anaesthesia with lidocaine and isoflurane combined with ketamine or fentanyl
}

\author{
Shi-Xia Zhang ${ }^{1,2}$, Xin $\mathrm{Li}^{2}$, Qing-Ming Ren², Dong-Liang $\mathrm{Niu}^{2}, \mathrm{Li} \mathrm{Gao}^{2}$, Hong-Bin Wang² \\ ${ }^{1}$ Agricultural University of Hebei, College of Veterinary Medicine, Baoding, P. R. China \\ ${ }^{2}$ Northeast Agricultural University, College of Veterinary Medicine, Harbin, P. R. China
}

Received November 4, 2014

Accepted November 10, 2015

\begin{abstract}
Fentanyl and ketamine are often used as adjuvants in intravenous anaesthesia to prolong analgesia. The aim of this study was to compare changes of the basic physiological variables of intravenous lidocaine administration in combination with ketamine or fentanyl, and to evaluate the impact of addition of fentanyl or ketamine to lidocaine on serum lidocaine concentrations in dogs after intravenous administration. During general anaesthesia, dogs of group L received $2 \%$ lidocaine intravenously, dogs of group LF received $2 \%$ lidocaine and fentanyl, and dogs of the group LK received $2 \%$ lidocaine and ketamine. The heart rate, systolic arterial pressure, diastolic arterial pressure, mean arterial pressure and rectal temperature decreased in all groups, and group LF showed the biggest effect on the basic physiological variables, with the lowest heart rate during the test, significantly decreased rectal temperature, and the most decreased values of arterial pressure. Blood for determination of serum lidocaine concentration was taken before anaesthesia and 5, 30, 60, 90, 120, 150 and $180 \mathrm{~min}$ after initial intravenous injection of drugs. Fentanyl and ketamine did not cause significant changes of serum lidocaine concentration in dogs and may be used as adjuvant in intravenous anaesthesia without a significant increase in lidocaine absorption.
\end{abstract}

Xylocaine, analgesia agent, cardiovascular system, canine

In small animal practice, inhalant drugs often cause dose-dependent cardiovascular depression. This situation, coupled with the fact that the analgesia provided by volatile anaesthetics is still controversial, justifies a continued search for improvement. Balanced anaesthesia which takes advantage of the well-known synergistic and additive interactions between injectable drugs (analgesics or sedatives) and volatile anaesthetics can decrease the dosages of each component while inducing general anaesthesia of sufficient depth with fewer side effects (Muir et al. 2003). Considering that volatile anaesthetics offer suboptimal analgesia, many authors focused on injectable agents that could provide sufficient analgesia to complement inhalant anaesthesia (Wilson et al. 2008). Thus, a combination of inhalant anaesthesia and analgesic has been widely advocated.

Lidocaine (L) is the commonly used perioperative analgesia agent for its $\mathrm{Na}^{+} / \mathrm{Ca}^{++}$ channel block function (Fozzard et al. 2005). Ketamine $(\mathrm{K})$ is a non-competitive NMDA receptor antagonist that has analgesic and anaesthetic properties and has been used to induce and supplement general anaesthesia (Wils on et al. 2008). Fentanyl (F) is a fast yet short-acting highly lipid soluble synthetic $\mu$-opioid (Pascoe 2000). It is frequently administered as an intravenous constant rate infusion (CRI) in the context of anaesthesia for perioperative analgesia and to reduce inhalant anaesthetic requirements (Ilkiw 1999). Therefore, in the present study, we established 3 anaesthesia protocols with either intravenous lidocaine alone or in combination with ketamine or fentanyl in dogs anaesthetized with isoflurane, comparing the changes in basic physiological variables.

Address for correspondence:

Hong-Bin Wang, PhD

College of Veterinary Medicine

Northeast Agricultural University

Harbin 150030, China
Phone: +86-451-55191833

Fax: +86-451-55190470

E-mail: hbwang1940@163.com

http://actavet.vfu.cz/ 
The toxic dose of serum lidocaine concentrations for muscle fasciculations is $8.21 \pm 1.69 \mu \mathrm{g} / \mathrm{ml}$ (Wallace et al. 1997). The animals were anaesthetized by intravenous use of lidocaine, therefore neurological signs could not develop. Serum lidocaine concentrations are worth detecting. In the present study, we used several analgesic drugs, but whether the complex interactions between the analgesic drugs would influence serum lidocaine concentrations was a question worth investigating. Song et al. (1994) and Gusak et al. (2013) found similar lidocaine plasma concentrations in the group which received epidurally lidocaine only and the group which received lidocaine with fentanyl or morphine. We are unaware of any literature on the influence of ketamine or fentanyl on lidocaine absorption from intravenous administration. Thus, the other purpose of this study was to evaluate the influence of ketamine or fentanyl addition to lidocaine on serum lidocaine concentrations in dogs after intravenous administration. Moreover, we aimed to evaluate the cardiovascular effects of the established three protocols which may have an influence on lidocaine absorption following intravenous administration and its serum concentrations in dogs anaesthetized with isoflurane.

\section{Materials and Methods}

Animals

The study was approved by the Animal Ethics Committee of the Northeast Agricultural University (20140306) in China. Eighteen mixed-breed adult dogs, male and female, weighing from 7 to $10 \mathrm{~kg}$, and 0.8 to 2 years of age, were used for this study. All the dogs were healthy - based on physical examination, complete blood (cell) count (CBC), serum biochemistry, and urinalysis.

\section{Experimental design}

Using a random numbers table, the dogs were assigned to three treatment groups, with each group consisting of six dogs. Staff and students working on the case were all blinded to the treatment given. The dogs of group $\mathrm{L}$ received intravenously lidocaine, loading doses (LD) of $3 \mathrm{mg} / \mathrm{kg}$, and then a constant rate infusion (CRI) of $3 \mathrm{mg} / \mathrm{kg} / \mathrm{h}$. The dogs of group LF received intravenously lidocaine (LD $3 \mathrm{mg} / \mathrm{kg} ; \mathrm{CRI} 3 \mathrm{mg} / \mathrm{kg} / \mathrm{h}$ ) and fentanyl (LD $0.004 \mathrm{mg} / \mathrm{kg}$; CRI $0.004 \mathrm{mg} / \mathrm{kg} / \mathrm{h}$ ). The dogs of group LK received intravenously lidocaine (LD $3 \mathrm{mg} / \mathrm{kg}$; CRI $3 \mathrm{mg} / \mathrm{kg} / \mathrm{h}$ ) and ketamine (LD $0.6 \mathrm{mg} / \mathrm{kg}$, CRI $0.6 \mathrm{mg} / \mathrm{kg} / \mathrm{h}$ ) (Aguado et al. 2011). Loading doses (LD) were made up with the calculated volume of $\mathrm{L}$ ( $2 \%$ lidocaine hydrochloride injection, Hualu Pharma Co., Ltd., Shandong, PR China), L+F (fentanyl, Shengyang Veterinary Drugs Pharma Co., Liaoning, PR China), or L $+\mathrm{K}$ (ketamine hydrochloride injection, Shengyang Veterinary Drugs Pharma Co., Liaoning, PR China); diluted to a final volume of $10 \mathrm{ml}$ in saline, and administered over $5 \mathrm{~min}$. Following LD administration, CRI was delivered using a syringe pump (Perfusor Secura FT; Braun, Netherlands). The CRI's were made up with the calculated volume of $\mathrm{L}, \mathrm{L}+\mathrm{F}$, or $\mathrm{L}+\mathrm{K}$ to final volumes of $20 \mathrm{ml}$ in saline and delivered at $10 \mathrm{ml}$ per hour for all groups. For each study group (L, LF, LK), solutions of intravenous drugs were prepared by a person not involved in the study.

\section{Pre-medication, induction and maintenance of anaesthesia}

Food, but not water, was withheld for $12 \mathrm{~h}$ before the experiment. The dogs were premedicated with atropine (atropine sulphate injection, Hainan Pharma Co., Ltd., Hainan, PR China) at a dose of $0.04 \mathrm{mg} / \mathrm{kg} \mathrm{BW} \mathrm{s.c.}$ Induction of anaesthesia was performed with propofol (propofol injection, Xian Libang Pharma Co., Ltd., Shanxi, PR China) at a dose of 4-5 mg/kg BW i.v. Following endotracheal intubation, anaesthesia was maintained with isoflurane (Isoflu, Jiupai Pharma Co., Ltd., Hebei, PR China) delivered through a circular breathing circuit. Intravenous anaesthesia was administered $30 \mathrm{~min}$ after the start of isoflurane administration.

The dogs were positioned in lateral recumbency. Ventilation volume and frequency were set to maintain normocarbia ( $\mathrm{etCO}_{2}=35-40 \mathrm{mmHg}$ ) before intravenous drugs administration, and isoflurane concentration was controlled until the end-tidal concentration reached $2.0 \%$. A body temperature probe was inserted into the rectum, and a heating blanket was provided to maintain body temperature. Anaesthetics were administered by the same staff.

Instrumentation, monitoring and data collection

Right or left cephalic vein was cannulated using a 22 or $24 \mathrm{G}$ over the needle catheter for the administration of the induction agent and lactated Ringer's solution at $10 \mathrm{ml} / \mathrm{kg}$ body weight (BW) per hour. A second IV catheter was placed for the administration of intravenous drugs.

The start of CRI was regarded as " 0 min", and CRI proceeded for $120 \mathrm{~min}$. After CRI, isoflurane inhalation anaesthesia was stopped. After withdrawal from isoflurane anaesthesia, the dogs were observed until the time 
of extubation and the first instance of standing upright was recorded. The extubation criteria were swallowing or signs of resistance to the endotracheal tube.

The electrocardiogram (lead II), heart rate (HR), systolic arterial blood pressure (SAP), diastolic arterial blood pressure (DAP) and mean arterial blood pressures (MAP), arterial haemoglobin saturation by oxygen $\left(\mathrm{SpO}_{2}\right)$ and rectal body temperature (RT) were monitored with a non-invasive monitor (Datex-OhmedaS/5TM; DatexOhmeda Division Instrumentarium) before anaesthesia, during anaesthesia (5, 10, 20, 30, 45, 60, 90 and 120 min after the start of the intravenous drugs CRI). Non-invasive blood pressure was monitored via a correctly sized cuff placed circumferentially around the left antebrachium of each dog, with the cuff width approximately 40 percent of the total circumference of the limb. Measurements were always taken by the same person and the median of 5 measurements was considered the final data.

Blood for determination of serum lidocaine concentration was collected in Li-heparin vials with a blood taking needle from the saphenous vein before general anaesthesia and 5, 30, 60, 90, 120,150 and 180 min after initial intravenous drug injection. Serum was separated by centrifugation $(3000 \times \mathrm{g}$ for $10 \mathrm{~min})$ and stored at $-80{ }^{\circ} \mathrm{C}$ and kept frozen until analysed. Lidocaine serum concentrations were determined using commercially available enzyme-linked immunosorbent assays for dogs (Beinglay Biotech Co., Ltd., Wuhan, PR China) as described by the manufacturer.

\section{Statistical analysis}

To validate the random allocation of the dogs into 3 groups, one-way ANOVA was used for continuous quantitative variables (age, weight) and for qualitative variable (sex). Cardiovascular indicators were analysed by means of ANOVA, during extubation and the first instances of standing upright after discontinuing isoflurane anaesthesia were also analysed. Data are reported as means \pm SD. The significance level was established as $P<0.05$. Statistical analysis of data was performed with the SPSS computer software (SPSS- Statistical Product and Service Solutions 13.0, SPSS Incorporation, Chicago, IL, USA).

\section{Results}

Table 1 shows the quantitative and qualitative variables of the dogs in the study. There were no significant differences, confirming that all dogs were randomly allocated to the L, LF and LK groups.

Table 1. Continuous quantitative and qualitative variables for dogs in groups L, LF and LK

\begin{tabular}{lccc}
\hline & Group L & Group LF & Group LK \\
\hline Age (years) & $1.55 \pm 0.54$ & $1.45 \pm 0.34$ & $1.53 \pm 0.45$ \\
Weight (kg) & $8.18 \pm 1.03$ & $8.72 \pm 0.91$ & $8.60 \pm 1.17$ \\
Sex \% (female/male) & $50.0 / 50.0$ & $66.7 / 33.3$ & $50.0 / 50.0$ \\
\hline
\end{tabular}

Group L - dogs received intravenously lidocaine; group LF - dogs received intravenously lidocaine and fentanyl; group LK - dogs received intravenously lidocaine and ketamine; $\mathrm{n}=6$ dogs per group. Data represent mean $\pm \mathrm{SD}$. No significant differences between groups were noted in any of the variables recorded.

The heart rate, rectal temperature, and arterial pressure during the 120 -min anaesthesia period in three groups of dogs are presented in Table 2. All three groups showed a reduction in the heart rate, but it remained within the general physiologic boundaries for the dogs in all 3 groups (70 to $130 \mathrm{bpm}$ ). The heart rate was $89.3 \mathrm{bpm}$ during LF anaesthesia, $111.2 \mathrm{bpm}$ during LK anaesthesia, and $105.0 \mathrm{bpm}$ during $\mathrm{L}$ anaesthesia (Table 2). There was a significant difference in the heart rate between groups LF and LK at 60 min $(P=0.024)$, and a significant difference between groups L and LF at $120 \mathrm{~min}(P=0.039)$. Within these three groups, there were no significant differences in the heart rate at the other time points.

Compared with the baseline rectal body temperature $\left(38.8 \pm 0.2^{\circ} \mathrm{C}\right)$, rectal temperature was decreased significantly during LF $\left(37.1 \pm 0.1{ }^{\circ} \mathrm{C} ; P=0.0005\right)$ and $\mathrm{LK}\left(37.7 \pm 0.8{ }^{\circ} \mathrm{C}\right.$; $P=0.016)$ anaesthesia. The rectal body temperature showed a decreasing trend during $\mathrm{L}$ anaesthesia $\left(38.3 \pm 0.4{ }^{\circ} \mathrm{C} ; P=0.117\right)$. There were significant differences in the rectal 
temperature between groups $\mathrm{L}$ and $\mathrm{LF}$ at $30 \mathrm{~min}(P=0.012), 60 \mathrm{~min}(P=0.005), 90 \mathrm{~min}$ $(P=0.003)$, and $120 \mathrm{~min}(P=0.031)$. The rectal temperature was significantly higher in group LK compared to group LF at $20 \mathrm{~min}(P=0.027), 60 \mathrm{~min}(P=0.0005)$, and $90 \mathrm{~min}$ $(P=0.016)$. There was a significant difference between $\mathrm{L}$ anaesthesia and LK anaesthesia

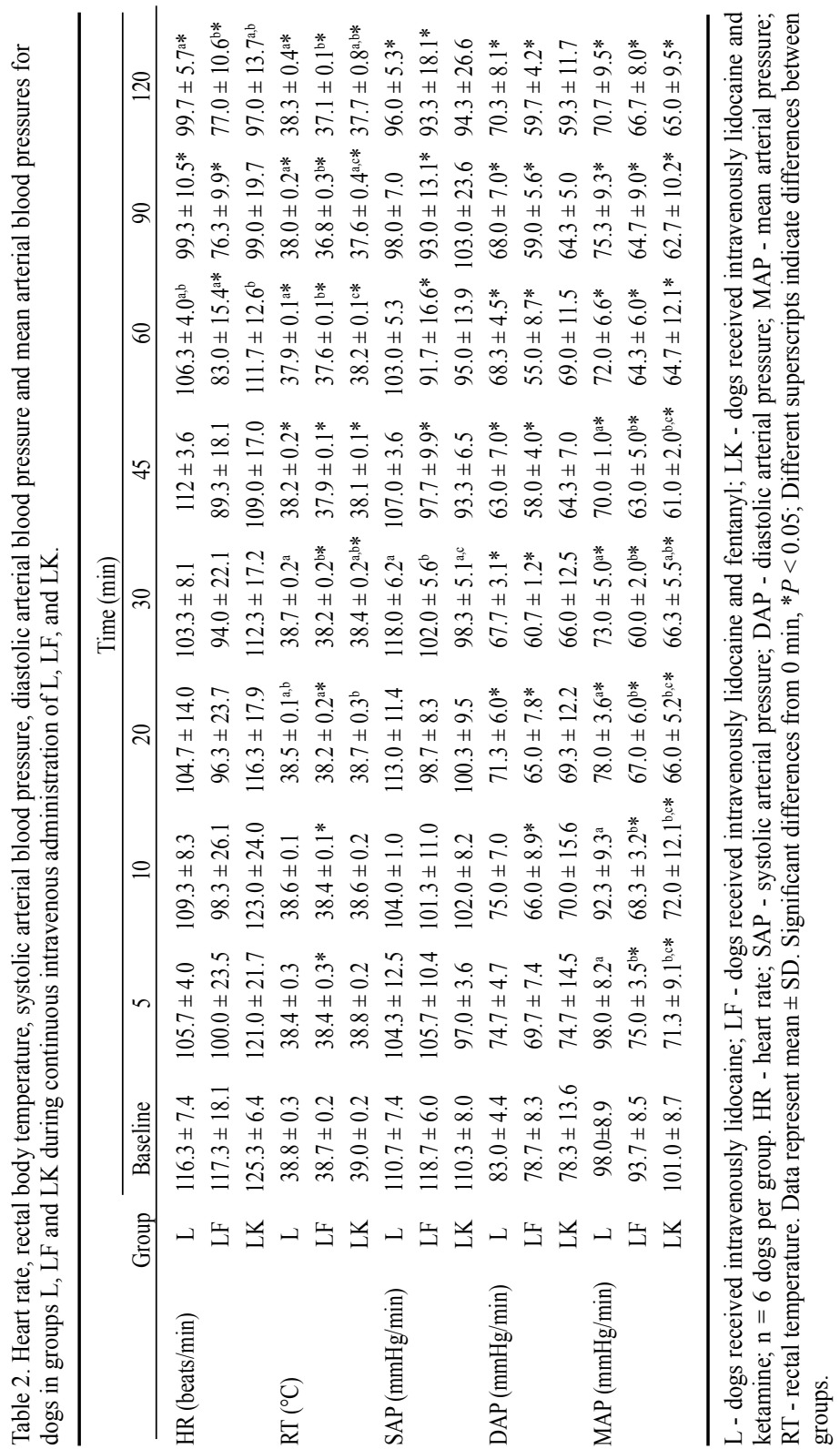

at $60 \min (P=0.005)$, and no significant difference at the other time points.

During the 120-min anaesthesia period in all three groups of dogs, group LF showed the most descended values of arterial pressures, with an observed marked decrease at 45-60 min. Hypotension with SAP below 90 $\mathrm{mmHg}$ and MAP below $60 \mathrm{mmHg}$ was not observed in any of the groups at any evaluated moment. The MAP decreased at 5 min in groups LF and $\mathrm{LK}$, and for up to $20 \mathrm{~min}$ for group $\mathrm{L}$ compared to $0 \mathrm{~min}$. The SAP increased in $\mathrm{L}$ anaesthesia for up to $20 \mathrm{~min}$ but keeping descended during LF and LK anaesthesia. Diastolic arterial pressure followed the values of MAP in all groups (Table 2). Systolic arterial pressure was significantly higher in group L compared to group LF at $30 \mathrm{~min}$ $(P=0.013)$. Within these three groups, there were no significant differences in diastolic arterial pressure at all time points.

Mean arterial pressure was significantly higher in group $\mathrm{L}$ compared to group LF at $5 \mathrm{~min}(P=0.009), 10 \mathrm{~min}(P=0.017), 20 \mathrm{~min}(P=0.037)$, 
$30 \min (P=0.012)$, and $45 \min (P=0.035)$. There were significant differences between group L and group LK at $5 \min (P=0.004), 10 \min (P=0.033), 20 \mathrm{~min}(P=0.027)$, and $45 \mathrm{~min}(P=0.013)$, and no significant difference between groups LF and LK all time points.

The duration from discontinuing isoflurane anaesthesia to extubation differed among all three groups. The shortest time was associated with group L and the longest with group LF. There were significant differences among the groups (LF compared with $\mathrm{L}, P=0.004$; LF compared with LK, $P=0.004$; Table 3 ). The duration between discontinuing inhaled anaesthesia and the first instance of standing also tended to be longer in group LF, and the difference among the groups was significant (LF compared to L, $P=0.012$; LF compared to $\mathrm{LK}, P=0.01)$.

Table 3. Duration of extubation and first instance of standing upright after discontinuing isoflurane anaesthesia.

\begin{tabular}{llcr}
\hline Groups & $\mathrm{n}$ & Time $(\min )$ extubation & FISU \\
\hline L & 6 & $6.3 \pm 4.2^{\mathrm{a}}$ & $18 \pm 8.9^{\mathrm{a}}$ \\
LF & 6 & $31.7 \pm 11.0^{\mathrm{b}}$ & $83.7 \pm 36.6^{\mathrm{b}}$ \\
LK & 6 & $6.3 \pm 2.3^{\mathrm{a}}$ & $15.0 \pm 9.6^{\mathrm{a}}$ \\
\hline
\end{tabular}

L - dogs received intravenously lidocaine; LF - dogs received intravenously lidocaine and fentanyl; LK - dogs received intravenously lidocaine and ketamine. FISU - first instance of standing upright. Data represent mean \pm SD. Different superscripts indicate differences between groups.

In all groups, lidocaine reached its peak concentration after $5 \mathrm{~min}$, the maximal serum concentrations of lidocaine were $1.34 \pm 1.31$ in group L, $1.94 \pm 1.75$ in group LF, and $1.49 \pm 1.50$ in group LK (means $\pm \mathrm{SD}$ ). However, the increase in the serum concentration of lidocaine in all groups was not particularly time-dependent. Group LF had the highest serum lidocaine concentration and group L had the lowest value after 5, 30 and $60 \mathrm{~min}$, but there was no significant difference between the groups. After 90 and $120 \mathrm{~min}$, minimal differences between groups were observed, but group LF had the lowest serum lidocaine concentration. Concentration at $180 \mathrm{~min}$ was about $50 \%$ value of the 5 min concentration in all groups, and a steady state was maintained after completing $120 \mathrm{~min}$ of CRI (Table 4).

Table 4. Serum lidocaine concentrations $(\mu \mathrm{g} / \mathrm{ml})$ for dogs in groups $\mathrm{L}, \mathrm{LF}$, and $\mathrm{LK}$ during and after continuous intravenous administration of $\mathrm{L}, \mathrm{LF}$, and LK.

\begin{tabular}{lcccccccc}
\hline Groups & \multicolumn{7}{c}{ Time (min) } \\
\cline { 2 - 9 } & 0 & 5 & 30 & 60 & 90 & 120 & 150 & 180 \\
\hline L & 0 & $1.34 \pm 1.31$ & $0.95 \pm 0.62$ & $0.73 \pm 0.47$ & $0.67 \pm 0.38$ & $0.80 \pm 0.51$ & $0.83 \pm 0.53$ & $0.73 \pm 0.54$ \\
LF & 0 & $1.94 \pm 1.75$ & $1.30 \pm 0.95$ & $1.12 \pm 1.21$ & $0.79 \pm 0.48$ & $0.71 \pm 0.38$ & $0.68 \pm 0.42$ & $0.86 \pm 0.57$ \\
LK & 0 & $1.49 \pm 1.50$ & $1.09 \pm 0.22$ & $0.79 \pm 0.47$ & $0.97 \pm 0.57$ & $0.82 \pm 0.62$ & $0.81 \pm 0.56$ & $0.90 \pm 0.53$ \\
\hline
\end{tabular}

L - dogs received intravenously lidocaine; LF - dogs received intravenously lidocaine and fentanyl; LK - dogs received intravenously lidocaine and ketamine; $\mathrm{n}=6$ dogs per group. Data represent mean $\pm \mathrm{SD}$. Significant differences from group $\mathrm{L}, * P<0.05$.

\section{Discussion}

During the 120-min anaesthesia, while dogs were warmed by a heating blanket, rectal body temperature was depressed compared to baseline values before anaesthesia. Dogs in groups LF and LK showed a lower rectal body temperature than those in group L. This result can be 
explained by the known influences of fentanyl and ketamine on thermoregulation (Ulugol et al. 2000; Zilberstein et al. 2008). Given that temperature changes of $2-3{ }^{\circ} \mathrm{C}$ are within the normal range, we consider the changes in the current study to be acceptable. Nevertheless, these findings underline the necessity of thermal support during anaesthetic procedures.

Fentanyl and ketamine are capable of producing multiple, at times intense, cardiovascular effects. The main effect is a decrease in the heart rate (Bhargava 1981) which corroborates the effect observed in the present study. This activity is due to the increase in systemic vascular resistance, which produces a compensatory bradycardia reflex mediated by baroreceptors (Gaynor and Muir 2008). In the present study, group LF presented a greater effect on the heart rate than group LK. Lidocaine can also cause decrease in $\mathrm{HR}$ as described by other literature reports for the depressive effect of lidocaine on the cardiovascular system (MacDougall et al. 2009). Although the heart rate in all three groups remained within the ranges considered to be normal for dogs, dogs anaesthetized with LF and LK displayed a lower heart rate than those anaesthetized with L alone.

Apart from causing changes in the heart rate, fentanyl and ketamine cause important changes in arterial pressure. Usually a decrease below basal values was observed, probably due to the vasodilatory effect (Almeida et al. 2007). Lidocaine is vasoconstrictor in subclinical doses, but clinical doses can cause vasodilatation, hypotension is the most frequent side effect of lidocaine administration (Gold et al. 1998). In the present study, a decrease in arterial pressures in all groups could be observed 5 min after the start of CRI and continued until the end of the experiment, and all values were within the physiological range. Group LF presented the greatest effect on arterial pressures compared to the other two groups in the present study.

Ketamine is widely used for intravenous induction and maintenance of general anaesthesia in dogs. It has analgesic properties even at subanaesthetic doses (Correll et al. 2004). Because recovery from ketamine is not smooth and muscle rigidity occurs, this drug is not recommended as the sole intravenous agent (Muir et al. 1977), even during inhalation anaesthesia (Muir and Sams 1992). Therefore, ketamine is commonly combined with other agents. Lidocaine has been successfully combined with ketamine in goats (Doherty et al. 2007) and dogs (Muir et al. 2003), resulting in reduction of additive isoflurane minimum alveolar concentration without ketamine-induced side effects. Fentanyl and other opioid analgesics have also been added recently to local anaesthetics to prolong postoperative analgesia.

Fentanyl and ketamine both have a vasodilatation effect, thus one of the goals of the present study was to confirm whether fentanyl and ketamine can be used intravenously without increasing serum lidocaine concentration in dogs. Song et al. (1994) found that lidocaine plasma concentrations were similar both in the group which received epidurally lidocaine only and in the group which received lidocaine with fentanyl. Gusak et al. (2013) found that fentanyl and morphine did not cause significant changes in serum lidocaine concentration in rabbits. In our study, the increase of lidocaine serum concentration in groups L, LF and LM was not significant at any time $(P>0.05)$. The highest serum concentration recorded in the groups at $5 \mathrm{~min}$ can be explained by possible histamineinduced vasodilatation and consequently by rapid lidocaine absorption.

About the recovery of lidocaine anaesthesia, some authors reported that epidural analgesia using lidocaine significantly slowed down recovery from isoflurane-induced anaesthesia in humans, and they thought that was because afferent nociceptive stimuli involved in promoting recovery from anaesthesia were blocked by epidural lidocaine (Inagaki et al. 1994). We found in the present study that the time until extubation and the first instance of standing upright tended to take longer in group LF compared to groups L and LK, but the differences were not significant. This might be because the vasodilatation effect of fentanyl and ketamine enhanced the recovery from lidocaine anaesthesia. 
In conclusion, the results of our study suggest that changes in the basic physiological variables after intravenous lidocaine administration in combination with ketamine or fentanyl were within the physiological range, and group LF showed the most marked effect, with the lowest HR during the test, significantly decreased BT, and the most decreased values of arterial pressures. Fentanyl and ketamine do not cause significant changes of serum lidocaine concentration in dogs and can be used as an adjuvant in intravenous anaesthesia in the tested doses.

\section{References}

Aguado D, Benito J, Gómez de Segura IA 2011: Reduction of the minimum alveolar concentration of isoflurane in dogs using a constant rate of infusion of lidocaine-ketamine in combination with either morphine or fentanyl. Vet J 189: 63-66

Almeida TF, Fantoni DT, Mastrocinque S, Tatarunas AC, Imagawa VH 2007: Epidural anesthesia with bupivacaine, bupivacaine and fentanyl, or bupivacaine and sufentanil during intravenous administration of propofol for ovariohysterectomy in dogs. J Am Vet Med Assoc 230: 45-51

Bhargava HN 1981: Antagonism of ketamine-induced anesthesia and hypothermia by thyrotropin releasing hormone and cyclo (His-Pro). Neuropharmacology 20: 699-702

Correll GE, Maleki J, Gracely EJ, Muir JJ, Harbut RE 2004: Subanesthetic ketamine infusion therapy: a retrospective analysis of a novel therapeutic approach to complex regional pain syndrome. Pain Med 5: $263-275$

Doherty T, Redua MA, Queiroz-Castro P, Egger C, Cox SK, Rohrbach BW 2007: Effect of intravenous lidocaine and ketamine on isoflurane minimum alveolar concentration in goats. Vet Anaesth Analg 34: 125-131

Fozzard HA, Lee PJ, Lipkind GM 2005: Mechanism of local anesthetic drug action on voltage-gated sodium channels. Curr Pharm Des 11: 2671-2686

Gaynor JS, Muir WW 2008: Handbook of Veterinary Pain Management. $2^{\text {nd }}$ edn. Philadelphia, Pennsylvania: Mosby, $672 \mathrm{p}$.

Gold MS, Reichling DB, Hampl KF, Drasner K, Levine JD 1998: Lidocaine toxicity in primary afferent neurons from the rat. J Pharmacol Exp Ther 285: 413-421

Gusak V, Turkovic V, Nesek-Adam V, Lerotic I, Popovic M, Brajenovic N, Brcic Karaconji I, Vnuk D 2013: Lidocaine serum concentration after epidural administration in combination with morphine and fentanyl in rabbit - A preliminary study. Res Vet Sci 94: 651-655

Ilkiw JE 1999: Balanced anesthetic techniques in dogs and cats. Clin Tech Small Anim Pract 14: 27-37

Inagaki Y, Mashimo T, Kuzukawa A, Tsuda Y, Yoshiya I 1994: Epidural lidocaine delays arousal from isoflurane anesthesia. Anesth Analg 79: 368-372

MacDougall LM, Hethey JA, Livingston A, Clark C, Shmon CL, Duke-Novakovski T 2009: Antinociceptive, cardiopulmonary, and sedative effects of five intravenous infusion rates of lidocaine in conscious dogs. Vet Anaesth Analg 36: 512-522

Muir WW 3rd, Wiese AJ, March PA 2003: Effects of morphine, lidocaine, ketamine, and morphine-lidocaineketamine drug combination on minimum alveolar concentration in dogs anesthetized with isoflurane. Am J Vet Res 64: 1155-1160

Muir WW, Sams R 1992: Effects of ketamine infusion on halothane minimal alveolar concentration in horses. Am J Vet Res 53: 1802-1806

Muir WW, Skarda RT, Milne DW 1977: Evaluation of xylazine and ketamine hydrochloride for anesthesia in horses. Am J Vet Res 38: 195-201

Pascoe PJ 2000: Opioid analgesics. Vet Clin North Am Small Anim Pract 30: 757-752

Song JJ, Han YJ, Lee JR, Song HS 1994: Dosage and plasma concentration of local anesthetics in epidural anesthesia for Caesarean section. Korean J Anesthesiol 27: 782-791

Ulugol A, Dost T, Dokmeci D, Akpolat M, Karadag CH, Dokmeci I 2000: Involvement of NMDA receptors and nitric oxide in the thermoregulatory effect of morphine in mice. J Neural Transm 107: 515-521

Wallace MS, Laitin S, Licht D, Yaksh TL 1997: Concentration effect relation for intravenous lidocaine infusions in human volunteers: effects on acute sensory threshold and capsaicin-evoked hyperpathia. Anesthesiology 86: $1262-1272$

Wilson J, Doherty TJ, Egger CM, Fidler A, Cox S, Rohrbach B 2008: Effects of intravenous lidocaine, ketamine, and the combination on the minimum alveolar concentration of sevoflurane in dogs. Vet Anaesth Analg 35: 289-296

Zilberstein LF, Moens YP, Leterrier E 2008: The effect of local anaesthesia on anaesthetic requirements for feline ovariectomy. Vet J 178: 214-218 